\title{
APPROXIMATION IN METRIC LINEAR SPACES
}

\author{
GUNTER ALBINUS \\ Academy of Sciences of G.D.R., Institute of Mathematics and Mechanics, \\ 108 Berlin, G.D.R.
}

\section{Introduction}

In this paper some questions of approximation theory in metric linear spaces are dealt with. We consider certain non-normable spaces like the space $C(\mathbf{R})$ of all continuous functions on the real line under the topology of uniform convergence on compact sets. A metric $d$ on $C(R)$ is defined in such a way that the subspaces $M_{m}$, $m=1,2, \ldots$, of polynomials of degree $<m$ are Cebyšev sets and there is a numerical method of calculating for any $f \in C(R)$ and any $m$ the unique polynomial $p_{m}$ satisfying $d\left(f, p_{m}\right)=\inf \left\{d(f, p): p \in M_{m}\right\}$ at least approximately to any prescribed accuracy. Because of the last fact it is hoped that practitians will pay attention to some special non-normable metric linear spaces and will suggest applications where a metric is a natural measure of the error while a norm is not suitable. This would undoubtelly stimulate further research on approximation in metric linear spaces.

The investigation of questions of approximation theory throws new light on metric linear spaces. It was from the approximation-theoretical point of view that new notions such as cylindrical semiquasinorms or norm-like quasinorms have been defined and large classes of metric linear spaces has been introduced such as the class of locally cylindrical spaces or the class of spaces which will be described in Section 1. It is possible that these definitions and classes will turn out to be useful also in connection with other questions than approximation-theoretical ones.

A metric linear space $(E, d)$ is a linear space $E$ together with a translationinvariant metric $d$, i.e.

$$
d(x+z, y+z)=d(x, y) \quad(x, y, z \in E),
$$

such that the linear operations are continuous with respect to $d$. We shall consider real linear spaces $E$ only. Let $\boldsymbol{R}$ denote the set of real numbers. The metric $\boldsymbol{d}$ generates a functional $|\cdot|$ on $E$ by $|x|:=d(x, 0)(x \in E)$. It has the following properties:

For all vectors $x, y \in E$ and for $t \in R$

$$
|x|=0 \Rightarrow x=0 \text {, }
$$


and

$$
0 \leqslant|x|=|-x|,
$$$$
|x+y| \leqslant|x|+|y|,
$$

$$
\lim _{t \rightarrow+0}|t x|=0
$$

and for each sequence $\left(x_{j}\right)$ in $E$ and each $t \in \boldsymbol{R}$

$$
\left|x_{j}\right| \rightarrow 0 \Rightarrow\left|t x_{j}\right| \rightarrow 0 \text {. }
$$

A functional $f$ on a real linear space $E$ which satisfies all of the conditions $\left(Q_{j}\right)$ $(j=0, \ldots, 4)$ will be called a quasinorm (several systems of notation are used; we follow [29]). Any quasinormed space $(E, f)$ is also a metric linear space $(E, f(\cdot-\cdot))$, and the term quasinormed space $(E,|\cdot|)$ will be used instead of metric linear space $(E, d)$.

Two examples of quasinorms in the real plane $E=R^{2}$, namely

$$
\begin{aligned}
|(x, y)|_{1}:=\frac{|x|+|y|}{1+|x|+2|y|} \text { and } \\
\qquad|(x, y)|_{2}:=\min \left\{\left(x^{2}+y^{2}\right)^{1 / 2}, 1+\exp \left[-\left(x^{2}+y^{2}\right)^{1 / 2}\right]\right\},
\end{aligned}
$$

show that the closed balls in a quasinormed space are not necessarily convex, bounded (in the topological sense), connected or similar to each other. Furthermore,

$$
|(2, y)|_{1}>\inf _{y^{\prime} \in R}\left|\left(2, y^{\prime}\right)\right|_{1}=\frac{1}{2},
$$

i.e. there is no point on the $y$-axis that is nearest to the point $(2,0)$.

\section{Proximinality of finite-dimensional subspaces}

A non-empty subset $A$ of a quasinormed space $(E,|\cdot|)$ is called proximinal if for each $x \in E$ there is at least one element $u_{0} \in A$ such that

$$
\left|x-u_{0}\right|=\inf _{u \in A}|x-u|=: d_{A}(x) .
$$

An element $u_{0} \in A$ which satisfies this equality is called the best approximation of $x$ in $A$. It easily follows by an argument of compactness that each finite-dimensional linear subspace in a normed space is proximinal. In the example $\left(\boldsymbol{R}^{2},|\cdot|_{1}\right)$ of the preceding section, however, the $y$-axis is a non-proximinal subspace, and the other space $\left(\boldsymbol{R}^{2},|\cdot|_{2}\right)$ contains no proper proximinal subspace at all. Thus it is a natural task to find classes of quasinormed spaces in which each finite-dimensional linear subspace is proximinal. Two such classes have been described in [3], [4], and [13]. We are going to define a class that contains both those classes.

Let $E$ be a real linear space. A functional $f$ on $E$ which satisfies the conditions $\left(\mathrm{Q}_{j}\right), j=1, \ldots, 4$, above is called a semiquasinorm. Let us call a semiquasinorm $f$ on $E$ cylindrical if for each $x \in E$ and all $s, t \in R$ the implication

$$
0<s \leqslant t \Rightarrow f(s x) \leqslant f(t x)
$$

holds and if there is an $\left.\left.f^{\infty} \in\right] 0,+\infty\right]$ such that for each $x \in E$

$$
\lim _{t \rightarrow+\infty} f(t x)=\left\{\begin{array}{lll}
0 & \text { if } & f(x)=0, \\
f^{\infty} & \text { if } & f(x)>0 .
\end{array}\right.
$$

The notion of a cylindrical semiquasinorm is justified by the fact that

$$
\{x \in E: f(x) \leqslant r\}=\{y+z: y, z \in E, f(y) \leqslant r \text {, and } f(z)=0\} .
$$

Let $(S, \Sigma, \mu)$ be a measure space such that there is at least one measurable set of positive finite measure, i.e. a set $A \in \Sigma$ with $0<\mu(A)<\infty$. Let $(\Phi(\cdot, s))_{s \in S}$ denote a family of cylindrical semiquasinorms on a real linear space $E_{0}$ such that for any non-zero vector $x$ the function $\Phi(x, \cdot)$ is measurable and different from 0 on a subset of a positive measure.

THEOREM 1. The set

$$
E:=\left\{x \in E_{0}:|x|:=\int \Phi(x, s) d \mu(s)<\infty\right\}
$$

is a linear subspace of $E_{0}$ and $|\cdot|$ is a quasinorm on $E$.

The conditions $\left(\mathrm{Q}_{j}\right), j=0, \ldots, 4$, are verified for $|\cdot|$ by a straightforward calculation. An example of such a space is the space $S(0,1)$ of the (equivalence classes of) Lebesgue-measurable functions (a.e. on $[0,1]$ equal to each other) on $[0,1]$ under the quasinorm .

$$
|x|=\int_{0}^{1} \frac{|x(t)|}{1+|x(t)|} d t .
$$

More generally, the class of $\Phi$-spaces described in [3], [11], or in [13] is contained in the class described in the theorem.

The proof of the proximinality of finite-dimensional subspaces in $\Phi$-spaces given in [3] can be used in the more general class, too.

THEOREM 2. Let $(E,|\cdot|)$ be the quasinormed space of the preceding theorem. Then each finite-dimensional linear subspace of $(E,|\cdot|)$ is proximinal.

Sketch of the proof. Let $M \subset E$ be an $m$-dimensional linear subspace, let $x \in$ $E \backslash M$, and let $\left(u_{j}\right)$ be a sequence in $M$ such that

$$
\left|x-u_{j}\right| \rightarrow d_{M}(x) .
$$

If $\left(u_{j}\right)$ contains a convergent subsequence, then its limit is a best approximation.

Thus let us assume that $\left(u_{j}\right)$ contains no convergent subsequence. Then there are $p \leqslant m$ linear independent $w_{1}, \ldots, w_{p} \in M$ and there is a subsequence $\left(u_{j_{k}}\right)$ such that $u_{j_{k}}=\lambda_{k 1} w_{1}+\ldots+\lambda_{k p} w_{p}+v_{k}$, where for each $q \in\{1, \ldots, p\}$ the sequence $\left(\lambda_{k q}\right)$ increases unboundedly and

$$
v_{k q}:=\frac{1}{\lambda_{k q}}\left(\lambda_{k, q+1} w_{q+1}+\ldots+\lambda_{k, p} w_{p}+v_{k}\right) \rightarrow 0
$$

and where $\left(v_{k}\right)$ is a convergent sequence in a complementary subspace $M_{1} \subset M$ of 
the $\operatorname{span}\left[w_{1}, \ldots, w_{p}\right]$. Now $S$ is decomposed into

$$
\begin{aligned}
A_{1}:=\left\{s \in S: \Phi\left(w_{1}, s\right)>0\right\}, \\
A_{q}:=\left\{s \in S: \Phi\left(w_{q}, s\right)>0=\Phi\left(w_{q-1}, s\right)=\ldots=\Phi\left(w_{1}, s\right)\right\}, \\
\\
q=2, \ldots, m,
\end{aligned}
$$

and

$$
A_{0}:=S \backslash\left(A_{1} \cup \ldots \cup A_{p}\right) .
$$

Then we get

$$
\left|x-u_{j_{k}}\right|=\sum_{q=1}^{p} \int_{A_{q}} \Phi\left[x-\lambda_{k q}\left(w_{q}+v_{k q}\right), s\right] d \mu(s)+\int_{A_{0}} \Phi\left(x-v_{k}, s\right) d \mu(s) .
$$

Using the cylindricity of the semiquasinorms $\Phi(\cdot, s)$ as well as the continuity of any semiquasinorm on finite-dimensional subspaces with respect to the norm topology, we get

$$
\lim _{k \rightarrow \infty} \Phi\left[x-\lambda_{k q}\left(w_{q}+v_{k q}\right), s\right]=\Phi^{\infty}(s) \quad\left(s \in A_{q}, q=1, \ldots, p\right)
$$

and

$$
\lim _{k \rightarrow \infty}\left|x-u_{j_{k}}\right|=\int_{A_{1} \cup \ldots \cup A_{p}} \Phi^{\infty}(s) d \mu(s)+\int_{A_{0}} \Phi\left(x-\lim v_{k}, s\right) d \mu \geqslant\left|x-\lim v_{k}\right|,
$$

i.e. $\lim v_{k}$ is the best approximation of $x$.

COROLLARY. Under the additional assumption that

$$
\int_{A} \Phi(u, s) d \mu(s)<\int_{A} \Phi^{\infty}(s) d \mu(s)
$$

holds for all measurable sets $A$ of positive measure and for all $u \in M$, we see from the proof that each sequence $\left(u_{j}\right)$ satisfying (1) contains a converging subsequence, i.e. $M$ is approximatively compact (see [10]) and the metric projection on $M$ has a property of continuity (see [24]).

A measurable subset $A$ of positive measure is called an atom if it contains no measurable subset $B$ such that $0<\mu(B)<\mu(A)$. The measurable functions $\Phi(x, \cdot)$ are constant a.e. on each atom $A$; thus their value on $A$ will be denoted by $\Phi_{A}(x)$. If an atom $A$ has infinite measure, then we have $\Phi_{A}(x)=0$ for all $x \in E$ and the atom can be omitted. Thus we shall assume that each atom $A$ has a positive finite measure $\mu_{A}$. In accordance with the integration theory, two atoms $A^{\prime}$ and $A^{\prime \prime}$ will be identified if $\left(A^{\prime} \cup A^{\prime \prime}\right) \backslash\left(A^{\prime} \cap A^{\prime \prime}\right)$ is a set of measure zero. If that is so, different atoms are disjoint sets. If the measure space $(S, \Sigma, \mu)=\left(\mu_{A}\right)_{A \in \mathcal{A}}$ is purely atomic and if

$$
\sum \Phi_{A}^{\infty} \mu_{A}<\infty,
$$

then $\mathscr{A}$ is a countable set and the space $(E,|\cdot|)$ in Theorem 1 is just a space of the class considered in [4]. The cylinders

$$
\left\{x \in E: \Phi_{A}(x)<\varepsilon\right\}, \quad A \in \mathscr{A}, \varepsilon>0
$$

form a basis of neighbourhoods of o in $E$. Thus we shall call these spaces locally cylindrical. Any metrizable locally convex space $\left(E,\left(p_{n}\right)\right)$ becomes under the usual quasinorm

$$
|\cdot|_{1}:=\sum_{n=1}^{\infty} 2^{-n} \frac{p_{n}}{1+p_{n}}
$$

a locally cylindrical quasinormed space.

We have seen that the spaces described by Theorem 1 form a large class of quasinormed spaces. It would be interesting to find metrizable topological linear spaces which are not isomorphic to a space or to a subspace of a space of this class. Another question is whether each metrizable topological space is isomorphic to a quasinormed space in which each finite-dimensional subspace is proximinal. The examples in the introduction suggest still another question: Does there exist for any finite-dimensional linear subspace $M$ in an arbitrary quasinormed space $(E,|\cdot|)$ a positive number $r>0$ such that for any $x \in E$ the inequality $d_{M}(x)<r$ implies the existence of a best approximation of $x$ in $M$ ?

\section{Norm-like quasinormed spaces}

Let us say that a subset $M$ of a quasinormed space $(E,|\cdot|)$ uniquely approximates if for each $x \in E$ there is at most one best approximation in $M$. The question whether a given linear subspace uniquely approximates can be rather difficult even in normed spaces. There is, however, a result due to M. G. Krejn which can be generalized to quasinormed spaces. In a real normed space $(E,\|\cdot\|)$, each (one-dimensional, finitedimensional) linear subspace approximates uniquely if and only if the unit ball is strictly convex, i.e. $\|x+y\|<2$ for any two different unit vectors $x, y$ in $E$. The second example in the introduction is a quasinormed space in which each linear subspace uniquely approximates but not all of its balls are strictly convex. The following theorem is due to A. I. Vasil'ev [26].

THEOREM 3. Let $(E,|\cdot|)$ be a real quasinormed space of a dimension $\geqslant 2$ such that there is an integer $n \geqslant 1$ such that for each $n$-dimensional subspace $M \subset E$ and each $r>0$ the balls $\{x \in M:|x| \leqslant r\}$ in $M$ are connected. Then the following statements with arbitrary $k, m \in\{1,2, \ldots, \operatorname{dim} E-1\}$ are equivalent to each other:

(i) each linear subspace approximates uniquely;

$\left(\mathrm{ii}_{k}\right)$ each $k$-dimensional linear subspace approximates uniquely;

(iii ${ }_{m}$ ) each m-dimensional linear subspace $M$ is a Čebyšev set, i.e. for each $x \in E$ there is exactly one best approximation in $M$;

(iv) for any pair $(x, y)$ of different vectors in $E$ the inequality $\left|\frac{1}{2}(x+y)\right|$ $<\max \{|x|,|y|\}$ holds.

The formulation of (iv) is due to Ahuja [1]. The connectedness assumption with $n=1$ in the theorem is equivalent to condition $\left(\mathrm{Q}_{5}\right)$ on $|\cdot|$. Under this assump- 
tion the equivalence of quite similar statements was proved in [2]. It is this result that has led to the following

Definition. A quasinorm $|\cdot|$ on a linear space $E$ is called norm-like if for each $x \in E \backslash\{0\}$ the function $t \mapsto|t x|$ on the positive real half-line is strictly monotone and if for each $r>0$ the ball

$$
K(0, r)=\{x \in E:|x| \leqslant r\}
$$

is convex.

THEOREM 4. Let E be a metrizable locally convex space whose topology is given by a sequence $\left(p_{n}\right)$ of seminorms. Then

$$
|x|:=\sup _{n} 2^{-n} \frac{p_{n}(x)}{1+p_{n}(x)} \quad(x \in E)
$$

defines a norm-like quasinorm on $E$ that also generates the topology of $E$.

The proof is not difficult at all and the theorem can be stated in the same way for the more general class of metrizable locally pseudoconvex spaces (see [4] or [22]). The quasinorm (3) reflects topological properties much better than (2) does. Furthermore, (3) is much easier to handle than (2). We have

$$
K(o, r)=\bigcap_{2^{n} r 1}\left\{x \in E: p_{n}(x) \leqslant \frac{2^{n} r}{1-2^{n} r}\right\} .
$$

The preceding theorem is the first step to answer the question whether there are non-normable quasinormed spaces satisfying condition (iv) in Theorem 3. An affirmative answer is given by

THEOREM 5. Let $E$ be a separable metrizable locally convex space. Then the topology of $E$ can be generated by a norm-like quasinorm whose balls are strictly convex if and only if there is a continuous norm on $E$.

Proof. The condition is obviously necessary, since a seminorm is a norm if its unit ball is strictly convex.

Let a continuous norm exist on $E$. Then the topology of $E$ can be described by a sequence of norms $\|\cdot\|_{1},\|\cdot\|_{2}, \ldots$ The separability of $E$ implies the separability of the normed spaces $\left(E,\|\cdot\|_{n}\right), n=1,2, \ldots$ It is well known that then there are norms $\|\cdot\|_{n}^{0}, n=1,2, \ldots$, on $E$ such that the unit balls of the norms $\|\cdot\|_{n}^{0}$ are strictly convex and such that for each $n$ the norms $\|\cdot\|_{n}$ and $\|\cdot\|_{n}^{0}$ are equivalent (see [9]). The quasinorm defined by $\left(\|\cdot\|_{n}^{\circ}\right)$ according to (3) satisfies (iv) in Theorem 3 , since its balls, as finite intersections of strictly convex sets, are strictly convex.

Problems of approximation become fairly simple in norm-like quasinormed spaces, since they can be reduced to problems in normed spaces. Each ball $K(\mathrm{o}, r)$, $r>0$, in a norm-like quasinormed space $(E,|\cdot|)$ is the unit ball of a seminorm $p^{(r)}$. Thus each norm-like quasinormed space $(E,|\cdot|)$ corresponds to a family of seminormed spaces $\left(E, p^{(r)}\right)$ or to a family of normed spaces $\left(E^{(r)},\|\cdot\|^{(r)}\right)$, where $E^{(r)}:=E / \operatorname{ker} p^{(r)}$ and $\|\cdot\|^{(r)}$ is the norm induced by $p^{(r)}$. In this way it becomes evident that each finite-dimensional subspace of a norm-like quasinormed space is proximinal.

EXAMPLE. Let $s$ be the space of all sequences $x=\left(\xi_{n}\right)$ of real or complex numbers. Under the norm-like quasinorm

$$
|x|:=\sup 2^{-n} \frac{\left|\xi_{n}\right|}{1+\left|\xi_{n}\right|}
$$

the space $(s,|\cdot|)$ has the remarkable property that each closed linear subspace is proximinal.

This can be proved by reduction to the finite-dimensional case, since for any closed linear subspace $M$ and any $x \in s \backslash M$ there is an integer $k$ such that

$$
|x-y| \geqslant d_{M}(x)>2^{-k-1} \quad(y \in M),
$$

i.e. the coordinates $\xi_{j}-\eta_{j}(j>k)$ of $x-y$ do not influence the value $|x-y|$. This example, in connection with R. C. James's characterization of reffexivity of Banach spaces by proximinality of their closed hyperplanes, has stimulated further investigations of proximinality of closed linear subspaces by $\mathrm{K}$. Floret and $\mathrm{M}$. Wriedt. It has turned out that the reffexivity of a complete metrizable locally convex space $E$ is only a necessary condition for the existence of a norm-like quasinorm on $E$ which generates the given topology and with respect to which each closed hyperplane is proximinal (see [12]). The following example has been taken from [28].

EXAMPLE. Let $E$ be a non-normable metrizable complete locally convex space whose topology is described by a sequence $\left(\|\cdot\|_{n}\right)$ of scalar product norms and let $|\cdot|$ denote the norm-like quasinorm defined by the $\|\cdot\|_{n}$ 's according to (3). Then the ball $K\left(\mathrm{o}, \frac{1}{4}\right)$ in $(E,|\cdot|)$ is just the unit ball of $\|\cdot\|_{1}$. The completion $E_{1}$ of the normed space $\left(E,\|\cdot\|_{1}\right)$ is different from $E$ because of the open mapping theorem. Let $x \in E_{1} \backslash E$. Then

$$
H:=\left\{y \in E:(y, x)_{1}=0\right\}
$$

is a closed non-proximinal hyperplane in $(E,|\cdot|)$. Let $E_{n}, n=1,2, \ldots$, denote the completion of $\left(E,\|\cdot\|_{n}\right)$. Then

$$
\left|\left(x_{n}\right)\right|_{\infty}:=\sup _{n} 2^{-n} \frac{\left\|x_{n}\right\|_{n}}{1+\left\|x_{n}\right\|_{n}}
$$

is a norm-like quasinorm on $\prod_{n=1}^{\infty} E_{n}$. Each closed hyperplane of the space $\left(\prod_{n=1}^{\infty} E_{n},|\cdot|_{\infty}\right)$ is proximinal, but the image of $H$ under the imbedding $y \rightarrow(y, y, \ldots)$ is a non-proximinal closed linear subspace.

This example shows another difference between Banach spaces and complete norm-like quasinormed spaces, since all closed linear subspaces of a Banach space are proximinal if all closed hyperplanes in it are proximinal. 


\section{A computational method}

The theoretical background of the method of computing best approximations in a finite-dimensional Cebyšev subspace is the following

THEOREM 6. Let $(E,|\cdot|)$ be a norm-like quasinormed space. A finite-dimensional subspace $M$ is a Čebyšev set if and only if $M$ is a Čbyšev set with respect to all the seminorms $p^{(r)}$, where $r \in\left\{d_{M}(x): x \in E \backslash M\right\}=: I_{M}$.

The proof is not difficult at all. If $M$ is a Cebyšev set, then the restrictions $p^{(r)} \mid M$ of the seminorms $p^{(r)}$ onto $M, r \in I_{M}$, must be norms. Therefore we have the following

COROLlary. A finite-dimensional linear Čebyšev subspace $M$ in a norm-like quasinormed space is approximatively compact, and thus the metric projection onto $M$ is continuous.

The approximative compactness follows from the approximative compactness of finite-dimensional subspaces in normed spaces and from the fact that in any finite-dimensional norm-like quasinormed space $(E,|\cdot|)$ (e.g. the space of $x$ and $M$ ) for each $r_{0}>0$ and for each $\lambda>1$ there is an $r>r_{0}$ such that $K(\mathrm{o}, r) \subset \lambda K\left(0, r_{0}\right)$ $=\left\{\lambda y: y \in K\left(0, r_{0}\right)\right\}$.

Remark. At the Conference on Approximation Theory held in Kaluga 1975 A. I. Vasil'ev announced a quasinorm in the real plane with respect to which the $x$-axis is a Cebyšev subspace but not an approximatively compact one.

EXAMPLE. For any real $p>1$ and for any integer $m \geqslant 1$ the set $M_{m}$ of polynomials of degree $<\mathrm{m}$ is a Cebyšev set in the space $L_{\mathrm{loo}}^{p}(R)$ under the quasinorm

$$
|f|=\sup _{n} 2^{-n} \frac{\|f\|_{L^{p}(-n, n)}}{1+\|f\|_{L^{p}(-n, n)}},
$$

since the condition in Theorem 6 is satisfied.

THEOREM 7. Let $M$ be a finite-dimensional linear Čebyšev subspace in a quasinormed space $(E,|\cdot|)$, where $|\cdot|$ is a norm-like quasinorm of type (3). If for each $r \in I_{M}=\left\{d_{M}(x): x \in E \backslash M\right\}$ there is a procedure $P_{r}$ of calculating for any $x \in E$ the best approximation of $x$ in $M$ with respect to the seminorm $p^{(r)}$ at least to any prescribed accuracy and if for $x \in E \backslash M$ there is a rough approximation $v_{0} \in M$ such that

$$
r_{0}:=\left|x-v_{0}\right| \in I_{M},
$$

then the following iteration method is convergent. Let $r_{k-1}$ be the number obtained in the $(k-1)$-th step; then $P_{r_{k-1}}$ is applied to calculate $v_{k} \in M$ such that

$$
p^{\left(r_{k-1}\right)}\left(x-v_{k}\right)<d_{M}^{\left(r_{k-1}\right)}(x)+\frac{2^{-k+1}}{r_{k-1}},
$$

where $d_{M}^{(r)}(x)=\inf \left\{p^{(r)}(x-v): v \in M\right\}$, and $r_{k}:=\min \left\{r_{k-1},\left|x-v_{k}\right|\right\}$.

The decreasing sequence $\left(r_{k}\right)$ converges to $d_{M}(x)$ and the sequence $\left(v_{k}\right)$ converges to the best approximation of $x$ in $M$.
For the proof see [6]. For quasinorms of type (3) the inclusion

$$
K(\mathrm{o}, s) \subset \frac{s}{t} K(\mathrm{o}, t)
$$

holds for any $0<s \leqslant t$. It plays an essential rôle in the proof. From (4) the estimate

is obtained.

$$
r d_{M}^{(r)}(x) \leqslant d_{M}(x) \quad\left(r \geqslant d_{M}(x)\right)
$$

\section{The space $C(R)$}

Let $C(R)$ denote the space of all real-valued or complex-valued continuous functions on the real line $R$. The topology of uniform convergence on the compact subsets of $\boldsymbol{R}$ is described by the sequence $\left(p_{n}\right)$ of seminorms $p_{n}$,

$$
p_{n}(f):=\max _{|t| \leqslant n}|f(t)|,
$$

or, equivalently, by the norm-like quasinorm (3). Concerning the existence, uniqueness, or characterization of best approximations in finite-dimensional subspaces, the quasinormed space $(C(R),|\cdot|)$ behaves very much like the Banach space $C(0,1)$ under the supremum norm. The quasinorm $|\cdot|$ on $C(R)$ corresponds to a family of weighted supremum seminorms

$$
p^{(r)}(f):=\max _{t \in R} \varphi_{r}(t)|f(t)|
$$

with weights

$$
\varphi_{r}(t)=\left\{\begin{array}{lll}
\frac{1}{2^{n} r}-1 & \text { if } & n-1<|t| \leqslant n \text { for each } n \text { satisfying } 2^{n} r<1, \\
0 & \text { if } & |t|>\max \left\{n: 2^{n} r<1\right\} .
\end{array}\right.
$$

Defining

$$
S_{f}:=\left\{t \in \boldsymbol{R}: \varphi_{|S|}(t)|f(t)|=1\right\} \quad(f \in C(R) \backslash\{0\}),
$$

we have the Kolmogorov criterion, namely

Theorem 8. A function $g$ in a linear subspace $M \subset C(R)$ is a best approximation of $f \in C(R) \backslash M$ if and only if the inequality

holds for each $h \in M$.

$$
\min _{t \in S_{S-a}} \operatorname{Re}[\overline{f(t)-g(t)}] h(t) \leqslant 0
$$

THEOREM 9. A finite-dimensional linear subspace $M$ is a Čebyšev set in $(C(R),|\cdot|)$ if and only if each function $g \in M \backslash\{0\}$ has at most $\operatorname{dim} M-1$ zeros.

THEOREM 10. Let $M$ be an m-dimensional linear Cebysev subspace $M$ in the space $(C(R),|\cdot|)$ of real-valued functions. Then $g \in M$ is the best approximation of $f \in C(R) \backslash M$ if and only if the set $S_{f-g}$ contains at least $m+1$ points $t_{0}<t_{1}<\ldots<t_{m}$ such that

$$
\operatorname{sgn}\left[f\left(t_{k-1}\right)-g\left(t_{k-1}\right)\right]=-\operatorname{sgn}\left[f\left(t_{k}\right)-g\left(t_{k}\right)\right] \quad(k=1,2, \ldots, m)
$$

holds. 
The proofs of all the three theorems are quite similar to the proofs of the corresponding theorems in $C(0,1)$ (cf. [18]). For any $m$ the subspace $M_{m}$ of polynomials of degree $<m$, in particular, satisfies the so called Haar condition in Theorem 9. Because of the alternation Theorem 10, the Remez algorithm can be adapted to the seminormed spaces $\left(C(R), p^{(r)}\right)$. In connection with Theorem 7 there is a computational method of calculating the best approximation approximately.

\section{Sequences of linear subspaces in quasinormed spaces}

For each function $f$ of the space $(C(\boldsymbol{R}),|\cdot|)$ a module of continuity can be defined by

$$
w_{f}(\delta):=\sup _{|h|<\delta}|f-f(\cdot-h)| \quad(\delta>0)
$$

in close analogy to the module of continuity of a continuous periodic function. A further analogy is the inequality of the Bernstein type

$$
\left|p^{\prime}\right| \leqslant 2 \operatorname{deg} p|p|
$$

for the derivation $p^{\prime}$ of a polynomial $p$. Thus one can ask about the connections between the modulus of continuity $w_{f}$ and the rate of convergence to zero of the distances $d_{n}(f)$ of $f$ from the subspaces of polynomials of degree $<n, n=1,2, \ldots$ It should be remarked, however, that the analogy to the periodic case is not too close. For instance, the quasinorm $|\cdot|$, unlike the supremum norm of a periodic function, is not invariant under translations $f \mapsto f(\cdot-h)$. A definite difference between the space $(C(R),|\cdot|)$ and the space of continuous $2 \pi$-periodic functions under the supremum norm appears in

THEOREM 12. Let $\left(g_{n}\right)$ be a sequence in $C(R)$ such that for each $n=1,2, \ldots$ the functions $g_{1}, \ldots, g_{n}$ are linearly independent and such that the linear space $D$ spanned by all $g_{n}$ is dense in $(C(R),|\cdot|)$. If there is a continuous seminorm on $C(R)$ whose restriction onto $D$ is a norm, then there is no sequence $\left(A_{j}\right)$ of continuous linear operators

$$
A_{j}: C(R) \rightarrow \operatorname{span}\left(g_{1}, \ldots, g_{n_{j}}\right)
$$

such that $\lim A_{j} f=f$ for all $f$ in $(C(R),|\cdot|)$.

In [6] for any given sequence $\left(A_{j}\right)$ a function $f_{0} \in C(R)$ has been constructed such that $\left(A_{j} f_{0}\right)$ does not converge to $f_{0}$. The functions $g_{n}(t)=t^{n-1}, n=1,2, \ldots$, satisfy the conditions on $g_{n}$ in the theorem. This result seems to be of some interest in connection with approximation processes in locally convex spaces. This topic has been taken up recently (see [8]).

A Jackson type theorem for measurable $2 \pi$-periodic functions $f$ on $\boldsymbol{R}$ such that

$$
|f|_{p}:=\int_{0}^{2 \pi}|f(x)|^{p} d x<\infty,
$$

where $p \in] 0,1\left[\right.$ is a fixed number, with respect to the quasinorm $|\cdot|_{p}$ was announced by V.I. Ivanov [14] at the Conference on Approximation Theory held in Kaluga
1975. He also pointed out that that the Jackson type theorem in which derivatives of $f$ appear does not hold in $L_{p}, 0<p<1$.

Let $\left(M_{j}\right)$ be a sequence of linear subspaces in a quasinormed space $(E,|\cdot|)$. The sequence is said to approximate rapidly if there is a decreasing null sequence $\left(a_{j}\right)$ of non-negative numbers such that

$$
d_{M j}(x)=O\left(a_{j}\right)
$$

for each $x \in M$; otherwise it is said to approximate slowly. Modifying a negative approximation theorem of Bernstein, H. S. Shapiro [23] has shown that each sequence of proper closed linear subspaces in a Banach space approximates slowly. His proof is based upon Baire's category theorem and can be generalized to the proof of

THEOREM 13. A sequence $\left(M_{j}\right)$ of linear subspaces in a complete quasinormed space $(E,|\cdot|)$ approximates rapidly if and only if

$$
\limsup _{j \rightarrow \infty} d_{M \in E}(x)=0 \text {. }
$$

Although the condition is formulated completely in metric terms, it is a topological linear property (see [5]). In [5] we can also find the proof of

THeOREM 14. The space $(s,|\cdot|)$ from the first example in Section 2 is, up to isomorphy, the only infinite-dimensional locally cylindrical quasinormed space in which there is a rapidly approximating sequence of finite-dimensional subspaces.

In a forthcoming paper [7] the following theorem will also be proved:

THEOREM 15. The space $(s,|\cdot|)$ is up to isomorphy the only infinite-dimensional $\Phi$-space in which there exists a rapidly approximating sequence of finite-dimensional subspaces.

Thus the question arises whether the existence of rapidly approximating sequences of finite-dimensional subspaces characterizes the space $s$ within the clas of complete quasinormed spaces introduced in Section 1 or even among all infinitedimensional complete quasinormed spaces.

\section{References}

[1] G.C. Ahuja, T. D. Narang, and Swaran Trehan, Best approximation on convex sets in a metric space, J. Approximation Theory 12 (1974) 1, p. 94.

[2] G. A 1 b in u s, Normartige Metriken auf metrisierbaren lokal konvexen topologischen Vektorräumen, Math. Nachr. 37 (1968), pp. 177-195.

[3] -, Approximative Kompaktheit in quasinormierten Funktionenräumen und Stetigkeitsuntersuchungen, ibid. 44 (1970), pp. 363-390.

[4] —, Uber lokal radialbeschränkte oder lokal pseudokonvexe metrisierbare Räume, ibid. 45 (1970), pp. 363-372.

[5] - Remarks on a theorem of S. N. Bernstein, Studia Math. 38 (1970), pp. 227-234.

[6] - Approximationstheorie im Raun $C(R)$, Beiträge zur Analysis 3 (1972), pp. 31-44.

[7] -, On the existence of rapidly approximating sequences of finite-dimensional subspaces in 
spaces of measurable functions, to appear in Colloq. Math Soc. J. Bolyai, 19. Fourier Analysis and Approximation Theory, Budapest 1976.

[8] M. B e c k e r, Linear approximation processes in locally convex spaces, Aequationes Math. 12 (1975), 1, p. 120.

[9] D. F. C u d i a, Rotundity, Proc. Sympos. Pure Math. Vol. VII, pp. 73-97, Providence, R. I., 1963.

[10] [N. V. Efimov, S. B. Stečkin] H. В. Ефимов, С. В. С те чи й Аnпроксимативная компактность и чебышевские множества, Докл. АНСССР 140 (1961), pp. 522-524.

[11] M. E hrli ch, Dualraum und Lokalbeschränktheit einer Klasse metrischer linearer Räume, Arch. Math. 26 (1975) 3, pp. 273-283.

[12] K. F1 o r e t und M. Wri edt, Reflexivität und Bestapproximation in Fréchet-Räumen, ibid. 23 (1972), pp. 70-72.

[13] [A. L. Garkavi] A. Л. Гаркав и, Теорема суиествования элемента наилучиего приближения в пространствах типа $(F)$ с интегральной метрикой, Мат. заметки 8 (1970), pp. 583-594. (English translation in Math. Notes 8 (1970), pp. 797-802.)

[14] [V. I. I va n о v] В. И. И в ано в, Tеорема Джексона в метрике $L_{p}$ для $0<p<1$, Конференция по теории приближения функций, Калуга 1975.

[15] R. C. J a mes, Characterisations of reflexivity, Studia Math. 23 (1964), pp. 205-216.

[16] P. Kos mol und M. Wriedt, Proximinalität und Algorithmen bei konvexer Approximation, Arch. Math. 26 (1975) 2, pp. 193-200.

[17] G. G. L o r e n t z, Approximation of functions, New York 1966.

[18] G. M e in a rdu s, Approximation von Funktionen und ihre numerische Behandlung, Berlin, Göttingen, Heidelberg 1964

[19] D. P a ll a s c h k e, Der Satz von Bernstein für lineare metrische Räume, Schriften der GDM.

[20] D. P a ll a s chke und G. P a n teli d is, Homotopieeigenschaften von Sphären in $\Phi$-Räumen und approximative Kompaktheit, Arch. Math. 20 (1969), pp. 176-185.

[21] G. Pantelidis, Approximationstheorie für metrische lineare Räme, Math. Ann. 184 (1969), pp. 30-48.

[22] S. R olewicz, Metric linear spaces, Warszawa 1972.

[23] H. S. S h a piro, Some negative theorems of approximation theory, Michigan Math. J. 11 (1964), pp. 211-217.

[24] I. S inge r, Some remarks on approximative compactness, Rev. Roumaine Math. Pure Appl. 9 (1964), pp. 167-177.

[25] —, Best approximation in normed linear spaces by elements of linear subspaces, Berlin, Göttingen, Heidelberg.

[26] [A. I. Vasil' e v] А. И. В а си ль е В, О структуре иаров и подрространствах единственности в линейном метрическом пространстве, Мат. заметки 13 (1973), pp. 541-550.

[27] -, В линейном метрическом пространстве чебышевская прямая не облзательно аппроксимативно компактна, Конференция по теории приближения функций, Калуга 1975.

[28] M. W r ie d t, Beiträge zur Approximationstheorie in metrisierbaren lokalkonvexen Räumen, Math. Nachr. 64 (1974), pp. 135-147.

[29] K. Y o s i d a, Functional analysis, Berlin, Göttingen, Heidelberg 1965.

Presented to the Semester

Approximation Theory

September 17-December 17, 1975
ANACH CENTER PUBLICATTONS, VOLUME 4

PWN-POLISH SCIENTIFIC PUBLISHERS

WARSAW 1979

INEQUALITIES FOR FRACTIONAL DERIVATIVES

ON THE HALF-LINE

\author{
V. V. ARESTOV
}

Institute of Mathematics and Mechanics, Sverdlovsk, U.S.S.R.

Let $n$ be integers, $S$ the real line $(-\infty, \infty)$ or the half-line $[0, \infty)$. Denote by $W_{n}(S)$ the set of functions $x \in C=C(S)$ such that $x^{(n)} \in L_{\infty}=L_{\infty}(S)$. It is known (G. H. Hardy, J. E. Littlewood [7], L. Neder [11]) that functions from $W_{n}(S)$ satisfy for $0<k<n$ the inequality

$$
\left\|x^{(k)}\right\|_{c} \leqslant G\|x\|_{c}^{1-k / n}\left\|x^{(n)}\right\|_{L_{\infty}}^{k / n}
$$

where $G=G(k, n, S)$. A. N. Kolmogorov [8] has calculated the least constant $G$ in (1.1) on the whole line $S=(-\infty, \infty)$. A corresponding result for the half-line is known only for $n=2$ (E. Landau [9], J. Hadamard [6]) and for $n=3$ (A. P. Matorin [10]); in particular, the Landau-Hadamard inequality has the form

$$
\left\|x^{\prime}\right\|_{c} \leqslant 2 \sqrt{\|x\|_{c}\left\|x^{\prime \prime}\right\|_{L_{\infty}}} .
$$

Estimates of the constant $G$ for $S=[0, \infty)$ were given in papers of A. Gornyr H. Cartan, A. P. Matorin, S. B. Stechkin and etc. (for references see [14]); pape. [12] of I. Schoenberg and Al. Cavaretta deals with the calculation of the best constant,

In this note inequality (1.1) is considered for derivatives of not necessarily integer order on the half-line $S=[0, \infty)$. The value of the least constant $G$ is calculated here for small $k, n$; in the general case lower and upper estimates for $G$ are given.

The notion of integrals and derivatives of arbitrary order has been introduced by J. Liouville, B. Riemann and in the periodic case by $\mathrm{H}$. Weyl. The subject has been considered by G. H. Hardy, J. E. Littlewood, H. Kober and others; detailed references may be found in $\mathrm{H}$. Bateman and A. Erdelyi [1].

One of the following two operators is called integral of order $\sigma>0$ :

$$
\begin{aligned}
& \left(J_{\sigma} y\right)(t)=\frac{1}{\Gamma(\sigma)} \int_{t}^{\infty}(\eta-t)^{\sigma-1} y(\eta) d \eta, \\
& \left(J_{\sigma}^{*} y\right)(t)=\frac{1}{\Gamma(\sigma)} \int_{0}^{t}(t-\eta)^{\sigma-1} y(\eta) d \eta .
\end{aligned}
$$

